\title{
Laboratory Testing for Middle East Respiratory Syndrome Coronavirus, California, USA, 2013-2014
}

\section{Mahtab Shahkarami, Cynthia Yen, Carol Glaser, Dongxiang Xia, James Watt, Debra A. Wadford}

Since Middle East respiratory syndrome coronavirus (MERSCoV) first emerged, the California Department of Public Health has coordinated efforts to identify possible cases in travelers to California, USA, from affected areas. During 2013-2014, the department investigated 54 travelers for MERS-CoV; none tested positive, but $32(62 \%)$ of 52 travelers with suspected MERS-CoV had other respiratory viruses.

$\mathrm{M}$ iddle East respiratory syndrome coronavirus (MERS$\mathrm{CoV}$ ) has been a global concern since its discovery in Saudi Arabia in 2012. As of April 29, 2015, >1,100 confirmed MERS cases and $>420$ associated deaths had occurred globally; all cases were linked to the Middle East (1). Importation of MERS-CoV by travelers from the Arabian Peninsula to regions outside the Middle East has been documented (2). In May 2014, the first 2 cases of MERS in the United States were identified in unrelated travelers from Saudi Arabia (3).

Each year, an estimated 16 million international travelers visit California (4), of whom 225,000 are visitors from the Middle East (5); thus, a risk exists for importation of MERS-CoV into California. Furthermore, global events such as the annual Hajj and Umrah pilgrimages draw 11,000 Americans to Saudi Arabia each year (6).

Because of the possible risk for disease transmission, the Centers for Disease Control and Prevention (CDC) and the World Health Organization have issued MERS-CoV travel advisories for pilgrims traveling to Saudi Arabia $(7,8)$. In the fall of 2012, the California Department of Public Health (CDPH) addressed the risk of MERS-CoV importation and convened a working group composed of clinicians, laboratory staff, infection control experts, emergency operations staff, and information officers. This working group regularly reviewed the CDC and World Health Organization updates, scientific publications, and laboratory logistics, and took steps at the state level to prepare for MERS. CDPH developed and disseminated guidance on surveillance, specimen collection for laboratory testing,

Author affiliation: California Department of Public Health,

Richmond, California, USA

DOI: http://dx.doi.org/10.3201/eid2109.150476 infection control, and contact tracing (9). A CDPH clinician was available around the clock 7 days a week to assist with individual suspected cases of MERS.

\section{The Study}

$\mathrm{CDPH}$ created a laboratory testing plan to detect or rule out MERS-CoV infection in patients who, after review by $\mathrm{CDPH}$ clinicians, met specific clinical and travel criteria, per CDC case definitions (10), to be considered a patient under investigation (PUI). Once a MERS PUI was identified, the patient's specimens were transported from the hospital or local public health laboratory to $\mathrm{CDPH}$ in Richmond, California, for MERS-CoV testing. Specimens tested for each PUI consisted of $\geq 1$ of the following: upper respiratory tract sample (nasopharyngeal and oropharyngeal swab specimens), lower respiratory tract sample (sputum and lower respiratory tract aspirates or washes), serum, or stool. Time from specimen collection to receipt at CDPH was up to 48 hours for most PUIs (37/52 [71\%]). Because subsequent steps in infection control and patient management heavily depended on the test results, MERS-CoV testing at CDPH was expedited; the typical turnaround time was 4 hours from receipt of specimens to reporting of results.

During February-June 2013, specimens from MERS PUIs were tested at CDPH for MERS-CoV by using an in-house real-time reverse transcription PCR (rRT-PCR) assay that amplified the following 3 targets in the MERSCoV genome: UpE, N2, and N3 (11). CDPH implemented CDC's Novel Coronavirus 2012 Real-Time RT-PCR Assay protocol subsequent to its Emergency Use Authorization by the US Food and Drug Administration in June 2013 (12).

For persons with a suspected past MERS-CoV infection, CDPH sent serum specimens to CDC for MERS-CoV serologic testing. Once MERS-CoV infection was ruled out, CDPH tested the remaining respiratory specimens from MERS PUIs for other respiratory pathogens. Specimens were tested by real-time PCR and rRT-PCR for the following agents (13): influenza A and B viruses, human metapneumovirus, respiratory syncytial virus, adenovirus, parainfluenza virus (types 1, 2, 3, and 4), enterovirus, rhinovirus, and Mycoplasma spp. If an adequate amount of specimen remained, specimens were also tested for the presence of human coronaviruses 229E, OC43, NL63, and HKU1 by rRT-PCR (13). 


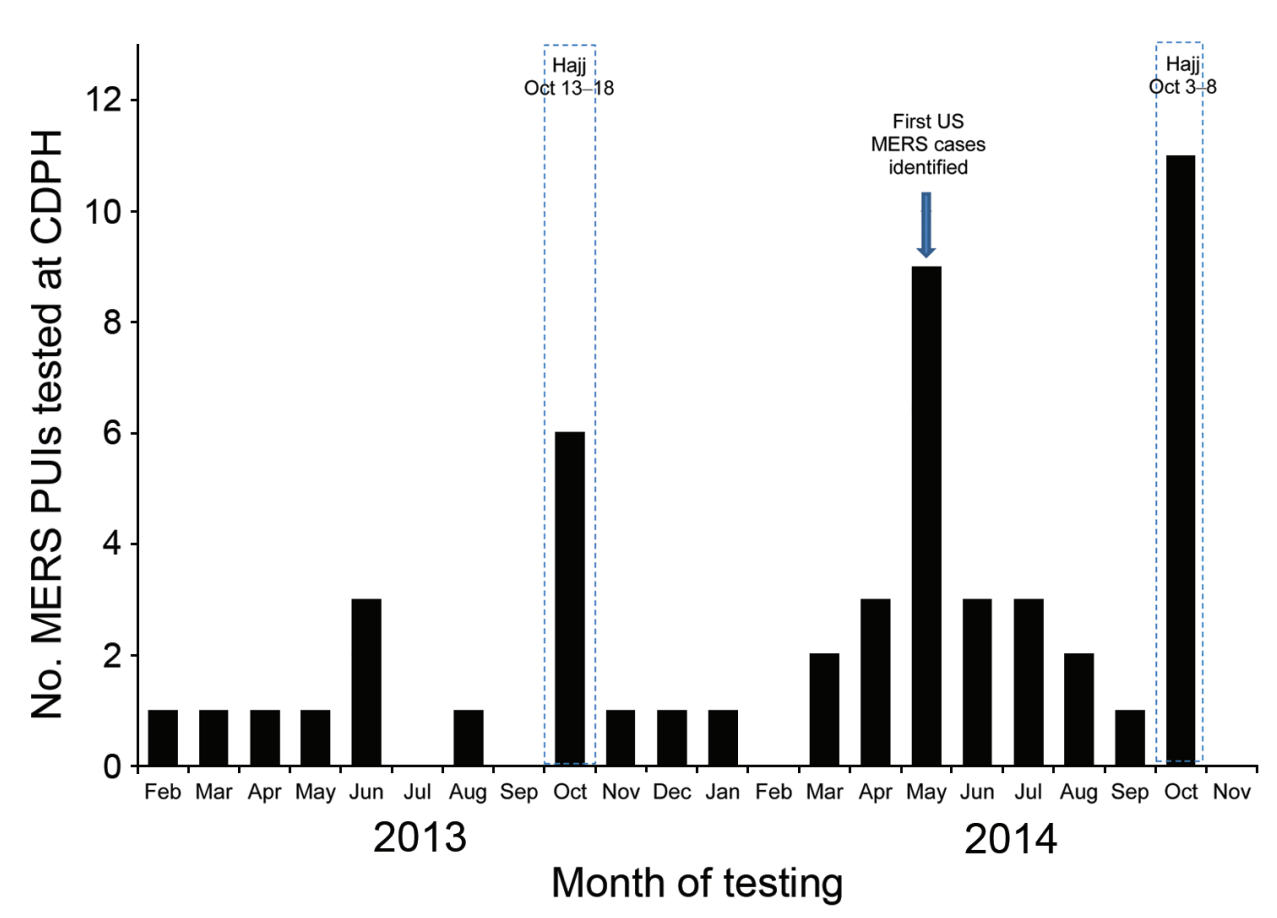

Figure. Middle East respiratory syndrome (MERS) coronavirus patients under investigation (PUIs) tested at the California Department of Public Health (CDPH), 2013-2014. Months during which the Hajj takes place are delineated by dashed lines.

During February 2013-November 2014, CDPH investigated 54 MERS PUIs in California, of whom 52 (total of 188 specimens) had testing conducted by CDPH and 2 had testing conducted by CDC (Figure). The median age for MERS PUIs was 53 years (range 10 months-89 years), and $57 \%$ were male and $43 \%$ female. A total of 51 (94\%) MERS PUIs reported travel from the Middle East, and $2(4 \%)$ were secondary contacts of travelers to the Middle East. A MERS PUI short form or equivalent was submitted to $\mathrm{CDC}$ and reported the following clinical outcomes for 42 MERS PUIs: 30 (71\%) hospitalized, $11(26 \%)$ admitted to an intensive care unit, $6(14 \%)$ intubated, $21(50 \%)$ received a diagnosis of pneumonia, and $5(12 \%)$ received a diagnosis of acute respiratory distress syndrome.

One or more respiratory viruses were detected in 32 (62\%) of the 52 MERS PUIs tested by CDPH; 5 of the 32 patients had a co-infection with rhinovirus plus another respiratory virus. Influenza, the most commonly identified respiratory agent, was detected in $18(35 \%)$ of the 52 MERS PUIs tested by CDPH (Table). Mycoplasma spp. was not detected in any specimen tested.

The frequency of MERS PUIs tested by CDPH varied with no apparent seasonality, except for the weeks following the Hajj in 2013 and 2014 (Figure). CDPH also noted an increase in reported MERS PUIs in May $2014(\mathrm{n}=9)$ after announcement of the first detected MERS cases in the United States (3). This increase likely resulted from media reports that heightened the level of concern among the public and health care workers, which increased the number of suspect MERS cases that CDPH and local partners had to evaluate for subsequent MERS-CoV testing.

\section{Conclusions}

As of May 7, 2015, MERS-CoV had not been detected in California. However, MERS-CoV poses a potential threat to global public health because MERS cases continue to be reported in Saudi Arabia, and the reservoir for the virus remains unclear, although camels have been implicated in disease transmission (14). CDPH has established a coordinated

\begin{tabular}{|c|c|c|}
\hline Virus detected & $\begin{array}{c}\text { No. patients, } \\
N=52\end{array}$ & $\begin{array}{c}\% \\
\text { Positive } \\
\end{array}$ \\
\hline Influenza only & 14 & 27 \\
\hline Influenza A (H3) & 10 & 19 \\
\hline Influenza $\mathrm{A}(\mathrm{H} 1 \mathrm{~N} 1)$ pdm09 & 3 & 6 \\
\hline Influenza B & 1 & 2 \\
\hline Noninfluenza only & 13 & 25 \\
\hline Respiratory syncytial virus & 1 & 2 \\
\hline Parainfluenza 3 & 2 & 4 \\
\hline Rhinovirus & 3 & 6 \\
\hline Enterovirus & 2 & 4 \\
\hline Human coronavirus $229 \mathrm{E}$ & 2 & 4 \\
\hline Adenovirus & 3 & 6 \\
\hline Co-infection & 5 & 10 \\
\hline Influenza $\mathrm{A}(\mathrm{H} 3)$ and rhinovirus & 1 & 2 \\
\hline $\begin{array}{l}\text { Influenza A(H1N1)pdm09 and } \\
\text { rhinovirus }\end{array}$ & 1 & 2 \\
\hline Influenza B and rhinovirus & 2 & 4 \\
\hline Parainfluenza 3 and rhinovirus & 1 & 2 \\
\hline No. patients with detected virus & 32 & 62 \\
\hline
\end{tabular}


statewide system working with local partners to identify potential MERS cases in California travelers returning from MERS-affected regions and their contacts. CDPH has investigated and conducted laboratory testing on $>50$ MERS PUIs and identified a respiratory virus in $62 \%$ of those patients, $35 \%$ of which were positive for influenza virus. The high rate of influenza detection underscores the need for all travelers to be immunized for influenza. CDPH continues to evaluate each MERS PUI and expedite MERS-CoV laboratory testing so that prompt implementation of containment procedures and contact investigations may proceed if needed.

\section{Acknowledgments}

We thank Tasha Padilla, Kara Pham, Estela Saguar, Ricardo Berumen III, Chao-Yang Pan, Hugo Guevara, Anthony Moore, Christopher Anderson, and Rosie Glenn-Finer for their technical and epidemiologic support. We also thank local health departments for their assistance in investigations and surveillance.

Ms. Shahkarami is a public health microbiologist specialist at the California Department of Public Health. Her main areas of work include molecular diagnostics and surveillance of respiratory viruses in California.

\section{References}

1. World Health Organization. Middle East respiratory syndrome coronavirus (MERS-CoV) - Saudi Arabia [cited 2015 May 7]. http://www.who.int/csr/don/29-april-2015-mers-saudi-arabia/en/

2. Centers for Disease Control and Prevention (CDC). Update: severe respiratory illness associated with Middle East respiratory syndrome coronavirus (MERS-CoV)-worldwide, 2012-2013. MMWR Morb Mortal Wkly Rep. 2013;62:480-3.

3. Bialek SR, Allen D, Alvarado-Ramy F, Arthur R, Balajee A, Bell D, et al. First confirmed cases of Middle East respiratory syndrome coronavirus (MERS-CoV) infection in the United States, updated information on the epidemiology of MERS-CoV infection, and guidance for the public, clinicians, and public health authorities - May 2014. MMWR Morb Mortal Wkly Rep. 2014;63:431-6.
4. Visit California. California statistics and trends [cited 2014 Aug 19]. http://industry.visitcalifornia.com/Find-Research/CaliforniaStatistics-Trends/

5. Visit California. Overseas and Mexican visitors to California, 2013 [cited 2015 May 11]. http://industry.visitcalifornia.com/ media/uploads/files/editor/CA\%20Intl\%20Report\%202013\%20 10-16-2014(1).pdf

6. Centers for Disease Control and Prevention. Hajj and Umrah in Saudi Arabia. 2015 [cited 2015 May 7]. http://wwwnc.cdc.gov/ travel/notices/alert/hajj-umrah-saudi-arabia-2015

7. World Health Organization. World - travel advice on MERS-CoV for pilgrimages [cited 2015 May 7]. http://www.who.int/ith/ updates/20130725/en/

8. Centers for Disease Control and Prevention. MERS in the Arabian Peninsula [cited 2015 May 7]. http://wwwnc.cdc.gov/travel/notices/ alert/coronavirus-arabian-peninsula-uk

9. California Department of Public Health. CDPH Middle East respiratory syndrome coronavirus (MERS-CoV) information webpage [cited 2015 May 11]. http://www.cdph.ca.gov/programs/ cder/Pages/MERS-CoV.aspx

10. Centers for Disease Control and Prevention. Middle East respiratory syndrome (MERS). Case definitions [cited 2015 Mar 1]. http://www.cdc.gov/coronavirus/mers/case-def.html

11. Lu X, Whitaker B, Sakthivel SK, Kamili S, Rose LE, Lowe L, et al. Real-time reverse transcription-PCR assay panel for Middle East respiratory syndrome coronavirus. J Clin Microbiol. 2014;52:6775. http://dx.doi.org/10.1128/JCM.02533-13

12. Centers for Disease Control and Prevention. Novel coronavirus 2012 real-time RT-PCR assay [cited 2015 May 11]. http://www.fda.gov/downloads/MedicalDevices/Safety/ EmergencySituations/UCM355572.pdf

13. Weinberg GA, Schnabel KC, Erdman DD, Prill MM, Iwane MK, Shelley LM, et al. Field evaluation of TaqMan Array Card (TAC) for the simultaneous detection of multiple respiratory viruses in children with acute respiratory infection. J Clin Virol. 2013;57:254-60. http://dx.doi.org/10.1016/j.jcv.2013.03.016

14. Adney DR, van Doremalen N, Brown VR, Bushmaker T, Scott D, de Wit E, et al. Replication and shedding of MERS-CoV in upper respiratory tract of inoculated dromedary camels. Emerg Infect Dis. 2014;20:1999-2005. http://dx.doi.org/10.3201/eid2012.141280.

Address for correspondence: Debra A. Wadford, California Department of Public Health, 850 Marina Bay Prkwy, Richmond, CA 94804, USA; email: Debra.Wadford@cdph.ca.gov

\section{The Public Health Image Library (PHIL)}

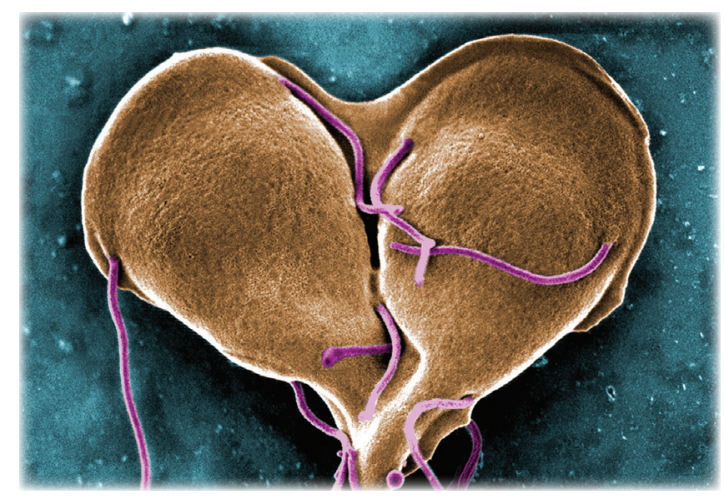

The Public Health Image Library (PHIL), Centers for Disease Control and Prevention, contains thousands of public health-related images, including high-resolution (print quality) photographs, illustrations, and videos.

PHIL collections illustrate current events and articles, supply visual content for health promotion brochures, document the effects of disease, and enhance instructional media.

PHIL Images, accessible to PC and Macintosh users, are in the public domain and available without charge.

Visit PHIL at http://phil.cdc.gov/phil 